\title{
Influence of Diffusion and Sorption on Sound Propagation in Multiscale Porous
} Materials

\author{
Rodolfo Venegas ${ }^{1}$, Claude Boutin ${ }^{2}$, and Olga Umnova ${ }^{3}$ \\ ${ }^{1}$ Université de Lyon - Ecole Nationale des Travaux Publics de l'Etat - LGCB/LTDS \\ - UMR-CNRS 5513, Rue Maurice Audin, 69518 Vaulx-en-Velin, France; \\ email: rodolfogustavo.venegascastillo@entpe.fr \\ ${ }^{2}$ Université de Lyon - Ecole Nationale des Travaux Publics de l'Etat - LGCB/LTDS \\ - UMR-CNRS 5513, Rue Maurice Audin, 69518 Vaulx-en-Velin, France; email: \\ claude.boutin@entpe.fr \\ ${ }^{3}$ Acoustics Research Centre, University of Salford, 43 Crescent, M5 4WT Salford, \\ United Kingdom; email: o.umnova@salford.ac.uk
}

\begin{abstract}
This paper investigates sound propagation in multiscale sorptive porous materials saturated with a pure gas. An example of this type of materials is a packing of porous grains where a mesoscopic, microscopic, and nanoscopic scale can be identified. These scales are associated with the grain size, and the pores inside the grains of micrometre and nanometre sizes, respectively. Considering that the inner-grain and inter-granular viscous permeabilities are highly contrasted, the macroscopic description of sound propagation through the material is established by upscaling a local mesoscopic description using the two-scale asymptotic method of homogenisation for periodic media. The local mesoscopic description is given by i) an homogenised description of the inner-grain physics that accounts for fluid flow and heat conduction in the micropores, inner-grain interscale mass difussion, and sorption occurring on the walls of the nanopores, ii) the equations describing fluid flow and heat conduction in the mesoscopic fluid network, and iii) the conditions of continuity of mass flux and pressure, and negligible temperature variations on the pore boundaries. It is concluded that the effective compressibility becomes significantly affected by sorption and interscale pressure and mass diffusion processes. This leads to a decrease in sound speed and an increase in sound attenuation, particularly around the characteristic frequencies associated to the diffusion processes.
\end{abstract}

\section{INTRODUCTION}

This paper investigates sound propagation in multiscale porous materials that support sorption and different types of diffusion processes. Sorption is a general term which refers to adsorption, desorption, and absorption (penetration of the fluid into the solid phase). The former is a physical or chemical process in which the fluid 
molecules are adhered onto a surface. Desorption is the opposite phenomenon, i.e., the fluid molecules are released from the surface (Do 1998). Adsorption/desorption is accompanied by diffusion that governs the flux of molecules from a region of higher concentration to one of lower concentration (Do 1998). An example of the materials investigated in this work is a packing of porous grains where a mesoscopic, microscopic, and nanoscopic scale can be identified, in addition to the macroscopic scale. These scales are associated with the grain size, and the pores inside the grains of micrometre and nanometre sizes, and to a characteristic size of the acoustic phenomena (i.e. sound wavelength), respectively. This paper extends the work (Venegas and Boutin 2017) to account for the influence of an additional mesoscopic scale that induces interscale (meso/micro-nano) pressure diffusion, in addition to the classical effects of heat conduction and viscosity at the introduced additional scale. The macroscopic acoustic description of sound propagation through multiscale sorptive porous materials is established in Section 2 using the two-scale asymptotic expansion method of homogenisation for periodic media (Auriault et al. 2009). The theory is exemplified for a packing of porous grains in Section 3. The main findings are summarised in the conclusions. This work was supported by the project METAUDIBLE (ANR 13-BS09-0003-03), cofunded by ANR and FRAE.

\section{THEORY}

\subsection{Geometry}

Consider a periodic multiscale sorptive rigid-frame porous material saturated with a pure Newtonian fluid. Figure 1 shows a diagram of the geometry of the material. The macroscopic characteristic length $L$ is related to the sound wavelength $\lambda$ through $L=|\lambda| / 2 \pi$. The representative elementary volume (REV) of the material is denoted as $\Omega=\Omega_{p f} \cup \Omega_{m n}$, where $\Omega_{p f}$ and $\Omega_{m n}$ are the volume of the pores (of surface $\Gamma_{p}$ ) and of the micro-nano porous domain. The latter has a REV $\Omega_{m}=\Omega_{m f} \cup \Omega_{n}$ where $\Omega_{m f}$ and $\Omega_{n}$ are the volume of the micropores (of surface $\Gamma_{m}$ ) and of the nano porous domain. The latter is composed of the volume $\Omega_{n f}$ of the nanopores (of surface $\Gamma_{n}$ ) and the volume of its solid part. The characteristic length associated with the pores (or the period of the material), micropores, and nanopores are denoted as $l_{p}, l_{m}$, and $l_{n}$, respectively; are well separated, i.e. $l_{n}<<l_{m}<<l_{p}$; and are usually milimetric, micrometric, and nanometric in size. Because of the separation of scales between $l_{p}$ and $l_{m}, \Omega_{m n}$ is modelled as an homogenised equivalent medium. Similarly, since $l_{n}<<l_{m}$, $\Omega_{n}$ is considered as an homogenised continuum governed by effective equations that are defined in the whole domain $\Omega_{n}$ and reflect the local physical processes. These correspond to volumetric diffusion of free gas molecules in the bulk of the nanopores and surface diffusion on the walls of the nanopores (see horizontal dashed lines in Figure 1). In addition to this, sorption occurs on the walls of the nanopores. The mass exchange between the gas (hollow circles) and adsorbate (black circles) phases is also depicted in Figure 1. The adsorbate volume is $\Omega_{s}=\Gamma_{n} N d$, where $d<l_{n}$ is the 


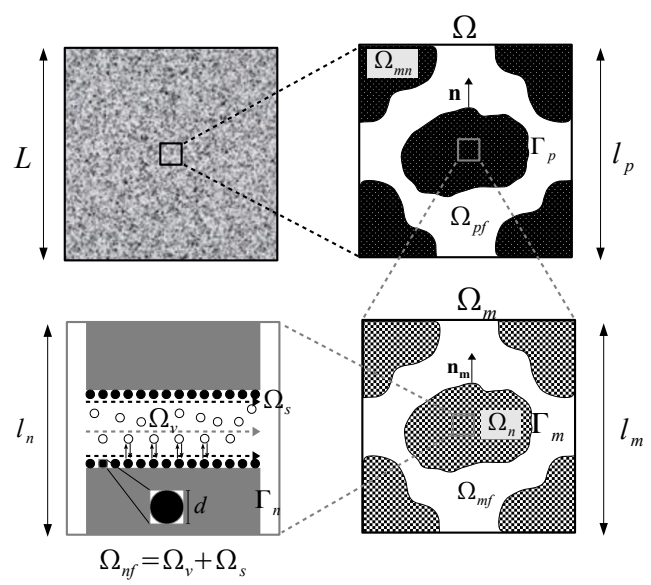

Figure 1. Diagram of the geometry of a multiscale sorptive porous material.

diameter of the molecules and $N=1$ is the number of adsorbed layers, i.e. monolayer coverage is assumed. $\Omega_{v}$ is the void space available for the transport of free molecules. The porosity of the material is $\phi_{p m n}=\phi_{p}+\left(1-\phi_{p}\right)\left(\phi_{m}+\left(1-\phi_{m}\right) \phi_{n}\right)$, where the porosities associated with the pores, micropores, and nanopores are $\phi_{p}=\Omega_{p f} / \Omega$, $\phi_{m}=\Omega_{m f} / \Omega_{m}$, and $\phi_{n}=\Omega_{n f} / \Omega_{n}$. The disparity in length scales between the pore size and the macroscopic characteristic size associated with the acoustic phenomenon provides a small expansion parameter $\varepsilon=l_{p} / L<<1$.

\subsection{Homogenisation procedure}

The scale separation $\varepsilon=l_{p} / L<<1$ allows applying the two-scale asymptotic expansion method of homogenisation for periodic media to establish an equivalent description from a mesoscopic re-scaled description, which is determined by the equations describing the physics in both the pore fluid network and the micro-nano porous domain upscaled to the pore-scale. The latter homogenised description has been established in (Venegas and Boutin 2017). Taking $L$ as reference length, the re-scaled equations in the pore fluid network are the linearised equations of conservation of momentum (1a), mass (1b), and energy (2a) and the equation of state (2b). The physical parameters involved are the dynamic viscosity $\eta$, specific heat capacity $C_{p}$, thermal conductivity $\kappa$. The oscillating velocity, pressure, density, and temperature are denoted as $\mathbf{u}_{p}, p_{p}, \rho_{p}$, and $\tau_{p}$, respectively. Note that the subscript 0 denotes an equilibrium quantity, and harmonic dependence of the type $e^{j \omega t}$ is assumed and, because of linearity, this term is omitted throughout the paper.

$$
\begin{gathered}
\varepsilon^{2} \eta \nabla^{2} \mathbf{u}_{p}-\nabla p_{p}=j \omega \rho_{0} \mathbf{u}_{p} \quad \text { and } \quad j \omega \rho_{p}+\rho_{0} \nabla \cdot \mathbf{u}_{p}=0 \quad \text { in } \quad \Omega_{p f}, \\
\varepsilon^{2} \kappa \nabla \cdot \nabla \tau_{p}=j \omega C_{p} \rho_{0} \tau_{p}-j \omega p_{p} \quad \text { and } \quad p_{p} P_{0}^{-1}=\rho_{p} \rho_{0}^{-1}+\tau_{p} \tau_{0}^{-1} \quad \text { in } \quad \Omega_{p f} .
\end{gathered}
$$

These equations are coupled to those describing the physics in the homogenised micronano porous domain via the following boundary conditions expressing the continuity 
of mass flux and pressure, and of negligible temperature variations on $\Gamma_{p}$ :

$$
\rho_{0} \mathbf{u}_{p} \cdot \mathbf{n}=\varepsilon \rho_{0} \mathbf{U}_{m} \cdot \mathbf{n} \quad ; \quad p_{p}=p_{m} \quad \text { and } \quad \tau_{p}=0 \quad \text { on } \quad \Gamma_{p} .
$$

Here $p_{m}$ represents the pressure in the micro-nano porous domain, while $\mathbf{U}_{m}$ the respective averaged velocity. The $\varepsilon-$ scaling, meaning that the advective mass flux pulsed by the micro-nano domain on $\Gamma_{p}$ is an order smaller than that in the pores, was estimated as $\mathcal{U}=\left|\rho_{0} \mathbf{U}_{m} \cdot \mathbf{n}\right| /\left|\rho_{0} \mathbf{u}_{p} \cdot \mathbf{n}\right|=\mathcal{K}_{m} p_{m} \eta L / \eta l_{p} l_{p}^{2} p_{p}$, where $\mathcal{K}_{m}=O\left(l_{m}^{2}\right)$ represents the viscous permeability of micropore fluid network. Using the continuity of pressure on $\Gamma_{p}$ one obtains $\mathcal{U}=l_{m}^{2} / l_{p}^{2} \varepsilon=O(\varepsilon)$, which holds as long as $l_{m} / l_{p}=O(\varepsilon)$.

The homogenised description of the micro-nano porous domain is given by:

$$
\varepsilon \nabla \cdot \mathbf{U}_{m}+j \omega p_{m} \mathbf{C}_{m n}(\omega)=0 \quad \text { and } \quad \eta \mathbf{U}_{m}=-\mathbf{k}_{m} \cdot \varepsilon \nabla p_{m} \quad \text { in } \quad \Omega_{m n},
$$

where the effective compressibility of the micro-nano domain is given by $C_{m n}(\omega)=$ $\mathrm{C}_{m}(\omega)+\mathrm{C}_{d s}(\omega)$, which depends on that of the micro porous domain $\mathrm{C}_{m}(\omega)=$ $\mathcal{Z}\left(P_{0}^{-1}, \phi_{m}, \omega(\gamma-1) / \omega_{t m} \gamma, \Theta_{m}(\omega) / \Theta_{m 0}\right)$ and of the nano porous domain $\mathrm{C}_{d s}=$ $\mathcal{Z}\left(P_{0}^{-1},\left(1-\phi_{m}\right) H_{e}, \omega / \omega_{d}, G(\omega) / G_{0}\right)$. Note that $\gamma$ is the adiabatic exponent and the function $\mathcal{Z}$ is given by Eq. (5). These effective compresibilities depend the thermal permeability of the micropore fluid network $\Theta_{m}(\omega)$, the interscale mass diffusion function $G(\omega)$ (Venegas and Boutin 2017), the static values of these two quantities, i.e. $\Theta_{m 0}$ and $G_{0}$; the thermal characteristic frequency associated to the micropore fluid network $\omega_{t m}=\phi_{m} \kappa / \rho_{0} C_{p} \Theta_{m 0}$; and the mass diffusion characteristic frequency $\omega_{d}=\left(1-\phi_{m}\right) \mathcal{D}_{a p p} / G_{0}$. The latter depends on the apparent effective diffusivity $\mathcal{D}_{a p p}=D_{e} / H_{e}$ with $D_{e}$ and $H_{e}$ being the effective diffusion coefficient and linearised effective sorption equilibrium constant, respectively.

$$
\mathcal{Z}(\mathcal{P}, \mathcal{Y}, \mathcal{W}, \mathcal{X})=\mathcal{P} \mathcal{Y}(1-j \mathcal{W} \mathcal{X})
$$

The physical variables are then looked for in the form of asymptotic expansions in powers of $\varepsilon$ as $Q(x, y)=\sum_{i=0}^{\infty} \varepsilon^{i} Q^{(i)}(x, y)$ where $Q=p_{p}, \mathbf{u}_{p}, \tau_{p}, \rho_{p}, p_{m}, \mathbf{U}_{m}$. These are then replaced in Eqs. (1)-(4) and the terms of the same order are identified. At $\varepsilon^{-1}$, it follows from Eq. (1a) that $\nabla_{y} p_{p}^{(0)}=0$, i.e. the pressure is a macroscopic variable $p_{p}^{(0)}=p_{p}^{(0)}(x)$. Further identification leads to the oscillatory Stokes and heat conduction problems in the pore fluid network with conditions of null velocity and null temperature on $\Gamma_{p}$. With $\overline{\mathbf{k}}_{p}(y, \omega)$ and $\bar{\theta}_{p}(y, \omega)$ representing the $\Omega_{p}$ - periodic local fields of velocity and temperature, their solutions read:

$$
\eta \mathbf{u}_{p}^{(0)}=-\overline{\mathbf{k}}_{p}(y, \omega) \cdot \nabla_{x} p_{p}^{(0)} \quad \text { and } \quad \kappa \tau_{p}^{(0)}=\bar{\theta}_{p}(y, \omega) j \omega p_{p}^{(0)},
$$

In the effective micro-nano porous domain, which is assumed isotropic for simplicity (i.e. $\mathbf{k}_{m}=\mathcal{K}_{m} \mathbf{I}$ with $\mathbf{I}$ being the unitary second-rank tensor), the local pressure field $p_{m}^{(0)}$ is governed by the following set (with $\mathcal{B}_{a p p}=\mathcal{K}_{m} / \eta \mathrm{C}_{m n}$ being the apparent pressure diffusivity):

$$
\nabla_{y} \cdot\left(\mathcal{B}_{a p p} \nabla_{y} p_{m}^{(0)}\right)=j \omega p_{m}^{(0)} \mathrm{C}_{m n} \quad \text { in } \quad \Omega_{m n} \quad \text { and } \quad p_{m}^{(0)}=p_{p}^{(0)} \quad \text { on } \quad \Gamma_{p} .
$$


This problem is formally identical to that of pressure diffusion in double porosity materials with highly contrasted permeabilities (Boutin et al. 1998). With $\bar{b}(y, \omega)$ representing the $\Omega_{p}$ - periodic local diffusive pressure field, the solution of the problem reads:

$$
p_{m}^{(0)}=p_{p}^{(0)}\left(1-j \omega \bar{b}(y, \omega) \mathcal{B}_{a p p}^{-1}\right)
$$

\subsection{Macroscopic acoustic description}

Integrating the leading-order mass balance equation over the pore volume, dividing by $\Omega$, and using the equation of state, Eq. (6b), and the thermodynamic identity $P_{0} / \tau_{0}=\rho_{0} C_{p}(\gamma-1) / \gamma$, lead to:

$$
j \omega p_{p}^{(0)} \mathrm{C}_{p}(\omega)+\nabla_{x} \cdot\left\langle\mathbf{u}_{p}^{(0)}\right\rangle+\left\langle\nabla_{y} \cdot \mathbf{u}_{p}^{(1)}\right\rangle=0,
$$

where the effective compressibility associated to the pore fluid network is calculated as $\mathrm{C}_{p}=\mathcal{Z}\left(P_{0}^{-1}, \phi_{p}, \omega(\gamma-1) / \omega_{t p} \gamma, \Theta_{p}(\omega) / \Theta_{p 0}\right)$. In this expression, the dynamic thermal permeability associated to the pore fluid network is given by $\Theta_{p}(\omega)=\left\langle\bar{\theta}_{p}(y, \omega)\right\rangle$,

with the averaging operator being defined as $\langle\cdot\rangle=\Omega^{-1} \int_{\Omega_{p f}} \cdot d \Omega$. The static value of the pore-fluid network thermal permeability is denoted by $\Theta_{p 0}$, while the mesoscopic thermal characteristic frequency reads $\omega_{t p}=\phi_{p} \kappa / \rho_{0} C_{p} \Theta_{p 0}$.

The third term in Eq. (9) is calculated by using the divergence theorem, noting that the integral vanishes on the periodic boundaries, and using the boundary condition Eq. (3a), Eq. (4a) at $\varepsilon^{0}$, and Eq. (8). This leads to the following macroscopic description:

$$
\eta\left\langle\mathbf{u}_{p}^{(0)}\right\rangle=-\mathbf{k}_{p}(\omega) \cdot \nabla_{x} p_{p}^{(0)} \quad \text { and } \quad \nabla_{x} \cdot\left\langle\mathbf{u}_{p}^{(0)}\right\rangle+j \omega p_{p}^{(0)} \mathrm{C}(\omega)=0,
$$

where the dynamic viscous permeability is calculated using Eq. (6) as $\mathbf{k}_{p}(\omega)=$ $\left\langle\overline{\mathbf{k}}_{p}(y, \omega)\right\rangle$ and the effective compressibility of the saturating fluid reads $\mathrm{C}(\omega)=$ $\mathrm{C}_{p}(\omega)+\mathrm{C}_{m n b}(\omega)$. Here $\mathrm{C}_{m n b}=\mathcal{Z}\left(\mathrm{C}_{m n},\left(1-\phi_{p}\right), \omega \mathcal{B}_{\text {app } 0} / \omega_{b} \mathcal{B}_{a p p}, B(\omega) / B_{0}\right)$ and the inter-scale pressure diffusion function is calculated as $B(\omega)=\Omega^{-1} \int_{\Omega_{m n}} \bar{b}(y, \omega) d \Omega$. The pressure diffusion characteristic frequency is given by $\omega_{b}=\left(1-\phi_{p}\right) \mathcal{B}_{\text {app } 0} / B_{0}$ with $\mathcal{B}_{\text {app } 0}=\mathcal{K}_{m 0} / \eta C_{m n 0}$ and $B_{0}=B(\omega=0)$. Note that $\mathcal{K}_{m 0}$ is the static viscous permeability associated to the micropore fluid network and the static effective compressibility of the micro-nano porous domain is given by $\mathrm{C}_{m n 0}=\left(\phi_{m}+\left(1-\phi_{m}\right) H_{e}\right) / P_{0}$. Further assuming that the material is isotropic, the dynamic viscous permeability becomes $\mathbf{k}_{p}=\mathcal{K}_{p} \mathbf{I}$. Then, the wave number $k_{c}$ and speed of sound $\mathcal{C}$ in the material are given by $k_{c}(\omega)=\omega \sqrt{\eta \mathrm{C} / j \omega \mathcal{K}_{p}}$ and $\mathcal{C}(\omega)=\omega / k_{c}(\omega)$.

\subsection{Analysis of the effective parameters}

The frequency behaviour of $\mathrm{C}(\omega)$ is determined by that of the thermal permeabilities and of the interscale mass and pressure diffusion functions. These take the following asymptotic values: $\Theta_{\iota}\left(\omega<<\omega_{t \iota}\right)=\Theta_{\iota 0}$ and $\Theta_{\iota}\left(\omega>>\omega_{t \iota}\right)=-j \phi_{\iota} \delta_{t}^{2}$ with $\iota=p, m ; G\left(\omega<<\omega_{d}\right)=G_{0}$ and $G\left(\omega>>\omega_{d}\right)=-j\left(1-\phi_{m}\right) \delta_{d}^{2}$; and 
$B\left(\omega<<\omega_{b}\right)=B_{0}$ and $B\left(\omega>>\omega_{b}\right)=-j\left(1-\phi_{p}\right) \delta_{b 0}^{2}$. In these expressions the thermal, mass diffusion and pressure diffusion boundary layer thickness are respectively given by $\delta_{t}=\sqrt{\kappa / \rho_{0} C_{p} \omega}, \delta_{d}=\sqrt{\mathcal{D}_{a p p} / \omega}$, and $\delta_{b 0}=\sqrt{\mathcal{B}_{a p p 0} / \omega}$. Then, the effective compressibility varies from $C_{l f}=\mathrm{C}\left(\omega<<\min \left(\omega_{t p}, \omega_{d}, \omega_{b}, \omega_{t m}\right)\right)$ to $C_{h f}=C\left(\omega>>\max \left(\omega_{t p}, \omega_{d}, \omega_{b}\right)\right)$. Only retaining linear terms in frequency, these limiting values are given by:

$$
\mathrm{C}_{l f}=P_{0}^{-1}\left(\phi_{p}+\left(1-\phi_{p}\right)\left(\phi_{m}+\left(1-\phi_{m}\right) H_{e}\right)-j \omega / \omega_{c}\right) \quad \text { and } \quad \mathrm{C}_{h f}=\phi_{p} / \gamma P_{0} \text {, }
$$

where $\omega_{c}$ is a global characteristic frequency that accounts for the thermal and diffusive processes in the material and is defined through :

$$
\frac{1}{\omega_{c}}=\frac{\gamma-1}{\gamma} \frac{\phi_{p}}{\omega_{t p}}+\left(1-\phi_{p}\right)\left(\frac{\gamma-1}{\gamma} \frac{\phi_{m}}{\omega_{t m}}+\frac{\phi_{m}}{\omega_{b}}\right)+\left(1-\phi_{p}\right)\left(1-\phi_{m}\right)\left(\frac{H_{e}}{\omega_{b}}+\frac{H_{e}}{\omega_{d}}\right) .
$$

It follows from Eq. (11) that the static compressibility is given by Eq. (13), which shows that, as a consequence of sorption, the low-frequency effective compressibility of the material can attain a value substantially larger than that of conventional porous materials. Such atypical compliance enhancement has been experimentally observed in activated carbon, e.g. (Venegas and Umnova 2011).

$$
\mathrm{C}_{0}=P_{0}^{-1}\left(\phi_{p}+\left(1-\phi_{p}\right)\left(\phi_{m}+\left(1-\phi_{m}\right) H_{e}\right)\right)
$$

On the other hand, the fluid flow at the leading order remains unaffected by the micronano porous domain physics (provided that $\mathcal{U}=O(\varepsilon)$ ). Considering leading-order terms only, the dynamic viscous permeability takes varies from $\mathcal{K}_{p}\left(\omega<<\omega_{v p}\right)=$ $\mathcal{K}_{p 0}$ to $\mathcal{K}_{p}\left(\omega>>\omega_{v p}\right)=-j \phi_{p} \delta_{v}^{2} / \alpha_{\infty}$; where $\omega_{v p}=\phi_{p} \eta / \rho_{0} \mathcal{K}_{p 0} \alpha_{\infty}$ is the viscous characteristic frequency, $\delta_{v}=\sqrt{\eta / \rho_{0} \omega}$ is the viscous boundary layer thickness, and $\alpha_{\infty}$ is the tortuosity. Hence, the following limiting values for the speed of sound are obtained:

$$
\mathcal{C}(\omega \rightarrow 0)=\sqrt{j \omega \mathcal{K}_{p 0} P_{0} / \eta \phi_{p m}\left(1+M_{H}\right)} \quad \text { and } \quad \mathcal{C}(\omega \rightarrow \infty)=c_{0} / \sqrt{\alpha_{\infty}}
$$

Reminding that $k_{c}(\omega \rightarrow 0)=\omega / \mathcal{C}(\omega \rightarrow 0)$, one concludes that low-fquency sound waves propagatin through multiscale sorptive materials are slowed down and more attenuated in comparison with non-sorptive ones. For example, by a factor of $\sqrt{1+M_{H}}$ when comparing to a double porosity non-sorptive material. At high frequencies, the influence of sorption and the inter-scale diffusion processes vanishes at the leading order.

\section{EXAMPLE}

A model for a packing of porous grains is used to exemplify the theory. The inner-grain structure is composed of two different scales of porosity. The smallest 
inner-grain scale corresponds to the nano porous domain. This is modelled as an effective medium with $H_{e}=\phi_{n}(\varphi+(1-\varphi) H)$, being $H$ the linearised sorption equilibrium constant that measures the local concentration of the adsorbed phase with respect to the gaseous one (Venegas and Boutin 2017); and $D_{e}=\phi_{n}\left(\varphi D_{n}+(1-\varphi) H D_{s}\right)$. The transport void fraction is approximated as $\varphi=1-d / r_{n}$, while the diffusion mechanism in the bulk of the nanopores is Knudsen diffusion, i.e. $D_{n}=D_{k}=2 r_{n} v_{T} / 3$ with $v_{T}$ being the mean thermal speed. The surface diffusion coefficient is guven by $D_{s}=(1 / 4) \zeta v_{T} \exp \left(-E_{a} / R_{g} \tau_{0}\right)$, where $R_{g}$ is the gas constant, $\zeta \approx d$ is the distance between adjacent sites, and $E_{a}$ is the energy of activation needed for a jump (Do 1998). The larger inner-grain scale is the micro porous domain $\Omega_{m}$. This is modelled as an array of cylindrical micropores with micropore radius $r_{m}$ and micro porosity $\phi_{m}$. The pore-scale geometry is modelled as an array of spherical grains with grain radius $r_{p}$ and inter-granular void porosity $\phi_{p}$. The effective parameters $\mathcal{K}_{m}, \Theta_{m}, \mathcal{K}_{p}, \Theta_{p}, G, B$ are calculated using Eqs. 74-76 and 84-86 in (Venegas and Boutin 2017). Consider the parameter values $r_{p}=0.5 \mathrm{~mm}, \phi_{p}=0.3, r_{m}=1 \mu \mathrm{m}, \phi_{m}=0.3, r_{n}=2.5 \mathrm{~nm}$ and $\phi_{n}=0.2, H=75$, and $E_{a}=10 \mathrm{~kJ} / \mathrm{mole}$; and that, for simplicity, the parameters of the saturating fluid are those of air $(d \approx 0.38 \mathrm{~nm})$ at normal pressure $\left(P_{0}=101325\right.$ $\mathrm{Pa})$ and temperature $\left(\tau_{0}=293.15 \mathrm{~K}\right)$ conditions.

Figure 2 shows the real and negative of the imaginary parts of the normalised dynamic effective compressibility of a triple-porosity sorptive material. This is compared with that of single, double, and triple porosity non-sortive materials, which respectively correspond to the cases of a packing of non-porous grains (i.e. $\phi_{n}=\phi_{m}=$ 0 ), and of porous grains without nanopores (i.e. $\phi_{n}=0$ ), and with $H_{e}=\phi_{n}$ and $D_{e}=\phi_{n} D_{k}$. Sorption induces an increase of the real part of the dynamic compressibility at low frequencies. For the four cases, the low-frequency limiting values are well predicted by Eq. (13), while at high frequencies the compressibility tends to Eq. (11b). On the other hand, multiscale sorptive materials provide larger sound attenuation than non-sorptive materials. This is a consequence of the combined effect of heat conduction in the pores and inter-scale mass and pressure diffusion, being the attenuation caused by the latter two enhanced by sorption. In particular, these processes lead to a decrease (increase) in low-frequency sound speed (attenuation), as shown in Figure 3.

\section{CONCLUSION}

The derived macroscopic description shows that the effective dynamic compressibility is significantly altered by the presence of the micro-nano porous domain. At the nanoscopic level, sorption increases the local density, slows down the mass diffusion, and modifies the pressure diffusion characteristics of the material. Macroscopically, these effects are manifested through an enhanced compliance of the effective saturating fluid and an increase (decrease) of the sound attenuation (speed of sound) in the material. The latter is particularly strong around the characteristic frequencies associated to the diffusion processes. 

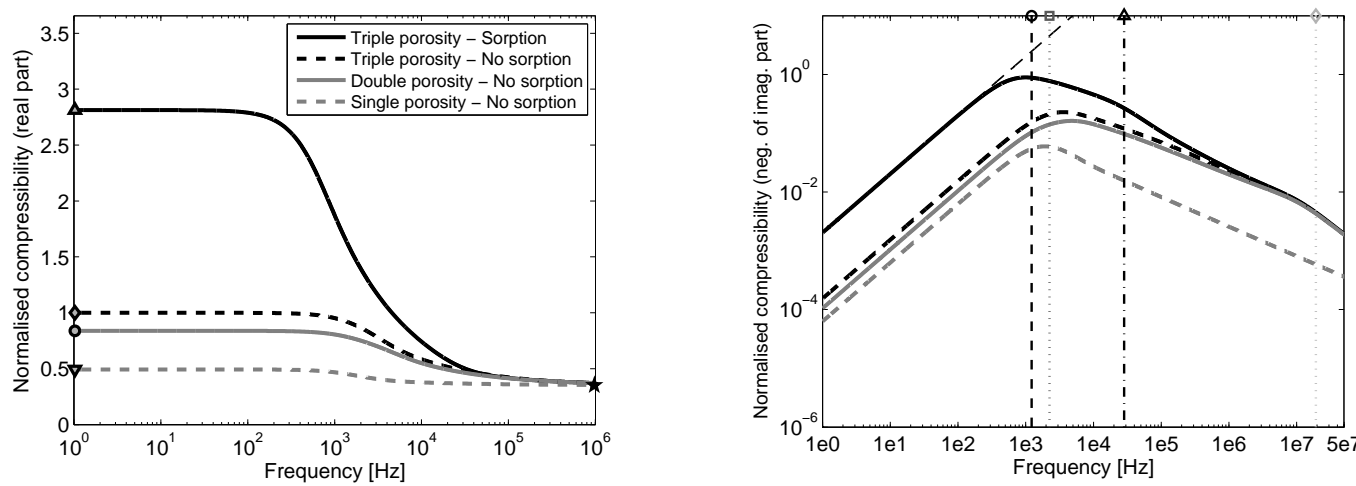

Figure 2. Left: Normalised real part of the effective dynamic compressibility $\operatorname{Re}\left(\mathrm{C}(\omega) P_{0} / \phi_{p m n}\right)$. Markers: limiting values. Right: $-\operatorname{Im}\left(\mathrm{C}(\omega) P_{0} / \phi_{p m n}\right)$. Vertical lines with a circle: $f_{b}$, square: $f_{t p}$, triangle: $f_{d}$, diamond: $f_{t m}$. Thin dashed black line: $-\operatorname{Im}\left(\mathrm{C}_{l f}(\omega) P_{0} / \phi_{p m n}\right)$ (Eq. (11)).
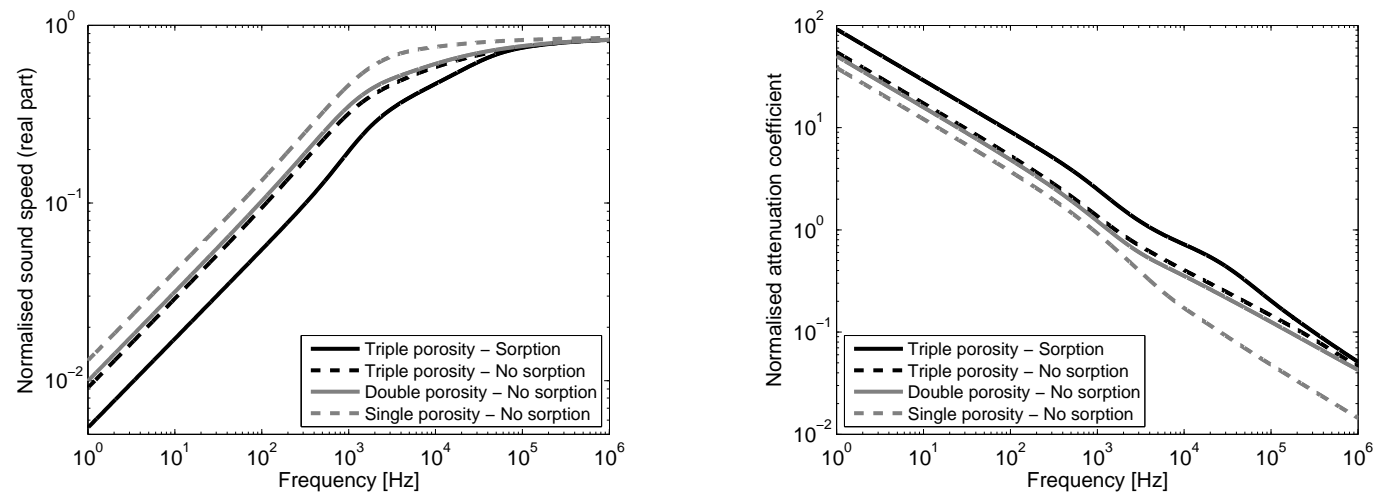

Figure 3. Left: Normalised speed of sound $\operatorname{Re}\left(\mathcal{C}(\omega) / c_{0}\right)$. Right: Normalised attenuation coefficient $:-\operatorname{Im}\left(k_{c}(\omega) c_{0} / \omega\right)$.

\section{REFERENCES}

Auriault, J.L., Boutin, C., Geindreau, C. (2009). Homogenization of Coupled Phenomena in Heterogeneous Media, ISTE Ltd and John Wiley \& Sons, London.

Boutin, C., Royer, P., Auriault, J.L. (1998). "Acoustic absorption of porous surfacing with dual porosity." Int. J. Solids Struct. 35 4709-4737.

Do, D. D. (1998). Adsorption Analysis: Equilibria and Kinetics, Imperial College Press, London.

Venegas, R., Umnova, O. (2011). "Acoustical properties of double porosity granular materials.” J. Acoust. Soc. Am. 130 (5), 2765-2776.

Venegas, R., Boutin, C. (2017). "Acoustics of sorptive porous materials.” Wave Motion $68,162-181$. 